EPJ Web of Conferences 60, 14002 (2013)

DOI: $10.1051 /$ epjconf $/ 20136014002$

(C) Owned by the authors, published by EDP Sciences, 2013

\title{
Measurements of vector bosons plus jets production with the ATLAS detector at the LHC
}

\author{
Lea Caminada ${ }^{1, a}$ on behalf of the ATLAS Collaboration \\ ${ }^{1}$ Lawrence Berkeley National Laboratory, 1 Cyclotron Road, Berkeley, CA 94720, USA
}

\begin{abstract}
The production of jets in association with a $W$ or $Z$ boson in proton-proton collisions at $\sqrt{s}=7 \mathrm{TeV}$ is measured with the ATLAS detector at the LHC. The cross sections, differential in several kinematic variables, have been measured up to high jet multiplicities and are compared to new higher-order QCD calculations. Measurements of vector bosons in association with heavy flavor, such as $W+c$ and $W+b$ production, have unique sensitivity to the heavy quark density of the proton. Differential cross sections are presented and compared to QCD predictions at NLO.
\end{abstract}

\section{Introduction}

Measurements of vector boson production in association with jets are important since they provide crucial information to improve our current understanding of strong interactions. The measurements test the predictions of perturbative QCD (pQCD) as well as their implementation in Monte Carlo (MC) simulations. Moreover, vector boson plus jets production presents a non-negligible background for studies of the Higgs bosons and other searches for new phenomena at the LHC and thus needs to be well understood.

High precision measurements of vector boson plus jets production are performed with the ATLAS experiment [1] at the LHC. The measurements use proton-proton collision data at a center-of-mass energy of $7 \mathrm{TeV}$ recorded by the ATLAS detector in 2010 and 2011. Cross sections of $W$ or $Z$ plus light jets production [2,3] are determined in events with high jet multiplicities and covering a large range of transverse momentum. Furthermore, $W+2$ jet events are analyzed to study double-parton interactions in proton-proton collisions [4]. Measurements of vector boson plus heavy flavor production, $W+b[5], W+c$ [6] and $Z+b$ [7], are performed to probe pQCD calculations in the presence of heavy quarks. These processes are also sensitive to the quark content of the proton and intended to improve the current knowledge of the parton distribution functions (PDF) to allow for more accurate cross-section predictions.

In the following the most recent measurements of vector boson plus jets production with the ATLAS experiment are presented.

\footnotetext{
ae-mail: lcaminada@lbl.gov
}

\section{$2 \mathrm{Z}+$ jets production}

The production of jets in association with a $Z$ boson is measured in data corresponding to an integrated luminosity of $4.6 \mathrm{fb}^{-1}$. The analysis is described in detail in Ref. [3]. Inclusive and differential cross sections are determined for jets with transverse momentum $p_{\mathrm{T}}>30 \mathrm{GeV}$ and rapidity $|y|<4.4$ in events with a $Z$ boson decaying leptonically to either electron or muon pairs. Cross sections are quoted in the fiducial phase space at particle level defined by the jets kinematics and the $Z$-boson kinematics (lepton $p_{\mathrm{T}}>20 \mathrm{GeV}$, lepton pseudorapidity $|\eta|<2.5$ and the two-lepton invariant mass in the range of $66 \mathrm{GeV}$ to $116 \mathrm{GeV})$.

The high-statistics data sets allow cross sections of $Z+$ jets production to be measured for multiplicities of up to seven jets as shown in Figure 1 (a). The total uncertainties on the inclusive cross sections range from $8 \%$ for $Z+1$ jet to $16-17 \%$ for $Z+\geq 4$ jets and are dominated by the uncertainty on the jet energy scale. The measurement is compared to fixed-order calculations at NLO pQCD computed using the BцаскHAT+SHERPA program [8-11]. These calculations are available up to $Z+4$ jets. Furthermore, the results are compared to predictions from different MC generators based on LO and NLO matrix elements supplemented by parton showers: ALPGEN [12] interfaced to HERWIG [13] for the modeling of the parton shower and to JIMMY [14] for the modeling of the underlying event, SHERPA using the MEnloPS approach [15] and MC@NLO [16] interfaced to HERWIG. Both ALPGEN and SHERPA employ matrix elements for up to five partons. MC@NLO generates the Drell-Yan process at NLO precision, which includes real emission of one additional parton. All higher parton multiplicities are generated by the parton shower. The MC samples are normalized globally to the NNLO inclusive $Z$ cross-section prediction [17]. 


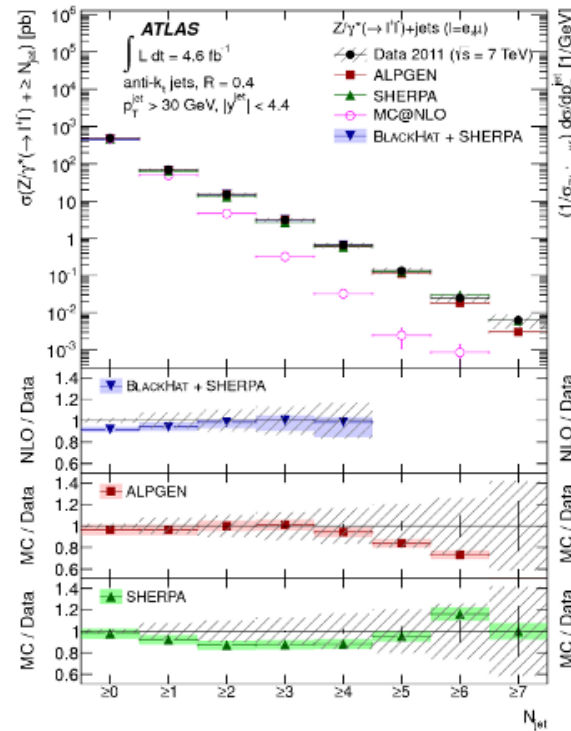

(a)

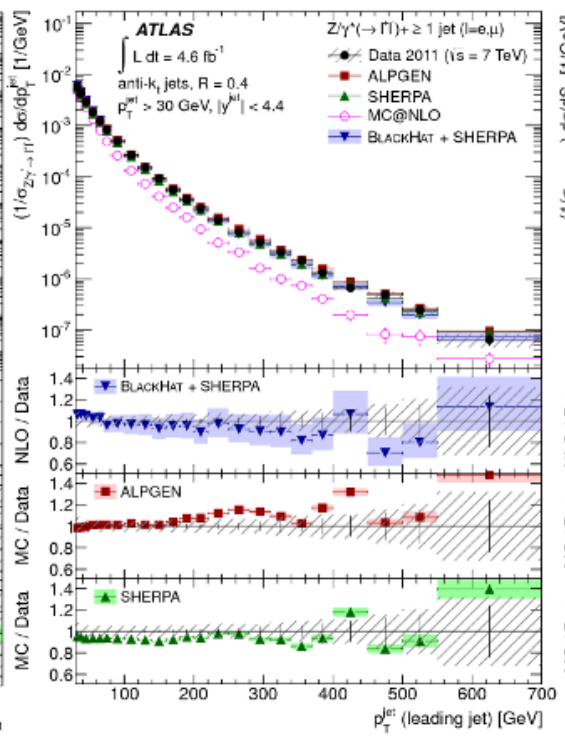

(b)

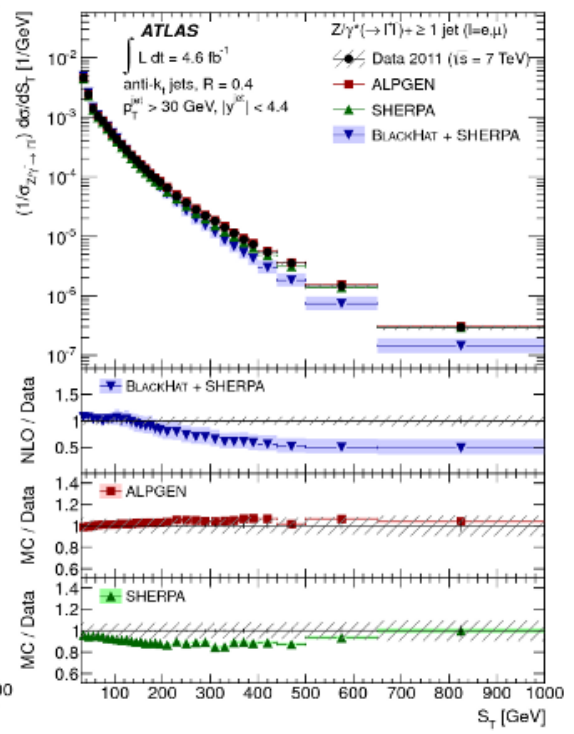

(c)

Figure 1: Measured cross section for $Z+$ jets production as a function of (a) the inclusive jet multiplicity, (b) the leading jet $p_{\mathrm{T}}$ and (c) the scalar $p_{\mathrm{T}}$ sum of the jets, $\mathrm{S}_{\mathrm{T}}$. The data are compared to NLO pQCD predictions from BLACKHAT+SHERPA corrected to the particle level, and the predictions of MC event generators. The error bars indicate the statistical uncertainty on the data, and the hatched (shaded) bands the statistical and systematic uncertainties on data (prediction) added in quadrature. The figures are taken from [3].

The measured cross sections as a function of the inclusive jet multiplicity are found to be consistent with the BLACKHAT+SHERPA calculation as well as with the predictions of ALPGEN and SHERPA. The MC@NLO parton shower underestimates the observed rate for additional jet emission, which leads to large offsets to the data for higher jet multiplicities.

Furthermore, the cross section is measured as a function of the jet $p_{\mathrm{T}}$ of the first, the second, the third and the fourth leading jet for events with at least one, two, three and four jets in the final state, respectively. As an example, the cross section as a function of the leading jet $p_{\mathrm{T}}$ is shown in Figure 1 (b). The cross sections are normalized to the inclusive $Z \rightarrow \ell \ell$ cross section to reduce the systematic uncertainties. The BцAскHAT+SHERPA prediction is in good agreement with data for all jet multiplicities. While the ALPGEN predictions for the jet $p_{\mathrm{T}}$ spectrum of the subleading jets are consistent with data, the predicted $p_{\mathrm{T}}$ spectrum of the leading jet is too hard for larger values of $p_{\mathrm{T}}$. The SHERPA prediction is characterized by a slight offsets to the data at the level of 5-15\%. MC@NLO predicts a too-soft jet $p_{\mathrm{T}}$ spectrum which is attributed to the fact that the fraction of events with a second resolved jet, which is modeled by the parton shower, increases considerably with jet $p_{\mathrm{T}}$. Accordingly, MC@NLO is found to provide a good description of the data if a veto on the second jet is applied.

Figure 1 (c) shows the cross section as a function of the inclusive quantity, $\mathrm{S}_{\mathrm{T}}$, the scalar sum $p_{\mathrm{T}}$ of the jets. ALPGEN provides a good description of the data, while the SHERPA prediction slightly underestimates the data, in line with the observation for the leading jet $p_{\mathrm{T}}$. The BLACK$\mathrm{H}_{\mathrm{AT}}+\mathrm{SHERPA}$ prediction deviates increasingly from the data for larger values of $S_{\mathrm{T}}$. The discrepancy is attributed to the missing higher jet multiplicities in the fixed-order calculation. The data is also compared to the exclusive sum of the $Z+1$-jet and $Z+\geq 2$-jets cross sections. The exclusive sum of the calculations provide a better description of the data which supports the assumption of missing higher order multiplicities in the inclusive calculation.

\section{DPI in $\mathrm{W}+2$ jets production}

The production of $W$ bosons in association with two jets has been analyzed for the presence of double-parton interactions (DPI) using data corresponding to an integrated luminosity of $36 \mathrm{pb}^{-1}$. In contrast to single parton interactions (SPI), in DPI events, the $W$ boson and the two jets are produced from different parton-parton interactions within the same proton-proton collision as illustrated in Figure 2.

The cross section for $W+2$ jets production in DPI events is described by an effective formalism as

$$
\hat{\sigma}_{W+2 \text { jets }}^{(\mathrm{DPI})}=\frac{\hat{\sigma}_{W} \cdot \hat{\sigma}_{2 \text { jets }}}{\sigma_{\text {eff }}}
$$

where $\sigma_{\text {eff }}$ is a parameter related to the proton size.

In this analysis, the fraction of $W+2$-jet events produced in DPI, $f^{(\mathrm{DPI})}$, is measured through the transverse momentum balance between the jets. The fraction of DPI events is then used to determine the value of $\sigma_{\text {eff }}$ using the 


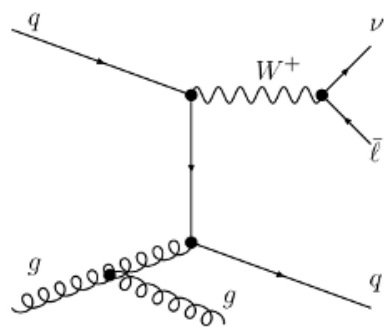

(a)

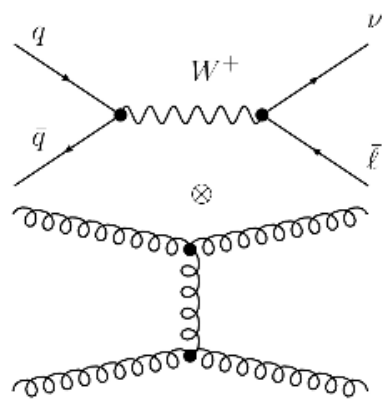

(b)

Figure 2: Leading-order Feynman diagrams for $W+2$ jets production in (a) single-parton interactions (SPI) and (b) double-parton interactions (DPI) [4].

following relation:

$$
\sigma_{\mathrm{eff}}=\frac{\sigma_{W_{0 j}} \cdot \sigma_{2 j}}{f^{(\mathrm{DPI})} \cdot \sigma_{W+2 j}}=\frac{1}{f^{(\mathrm{DPI})} \cdot \mathcal{L}} \cdot \frac{N_{W_{0 j}} \cdot N_{2 j}}{N_{W+2 j}}
$$

where $\mathcal{L}$ is the integrated luminosity, $N_{W_{0 j}}$ the number of $W+0$-jet events, $N_{2 j}$ the number of 2-jet events and $N_{W+2 j}$ the number of $W+2$-jet events. This relation holds because acceptance and efficiency correction factors cancel in the ratio.

The fraction, $f^{(\mathrm{DPI})}$, is extracted by a template fit to the distribution of the transverse momentum balance which provides a good discriminating power between SPI events and the back-to-back topology of jets in $W+2$-jet DPI events. The SPI template is obtained from simulation while a data-driven template from dijet events is used to describe the shape of the distribution in DPI events. The fits results in a fraction of DPI events of

$$
f^{(\mathrm{DPI})}=0.08 \pm 0.01(\text { stat }) \pm 0.02(\text { syst }) .
$$

The total systematic uncertainty on the measurement of $f^{\text {(DPI) }}$ is $24 \%$ where theory uncertainties and experimental uncertainties due to pile-up, jet energy scale and resolution and background modeling contribute to a similar amount. The above result leads to a measured central value of

$$
\sigma_{\text {eff }}=15 \pm 3 \text { (stat) }{ }_{-3}^{+5} \text { (syst) } \mathrm{mb} .
$$

This value is consistent with values previously measured in other experiments at lower center-of-mass energies. More details about this measurement are presented in Ref. [4].

\section{$4 W+b$}

A measurement of the cross section for $W$-boson production in association with $b$-quark jets is performed using data corresponding to an integrated luminosity of $4.6 \mathrm{fb}^{-1}$. The measurement is performed differentially in the $b$-jet transverse momentum for the 1-jet and 2-jet final states.

The $W+b$-jets cross section is measured in a restricted fiducial region: Events at the generator level are required to contain an electron or a muon with $p_{\mathrm{T}}>25 \mathrm{GeV}$ and $|\eta|<2.5$ and a neutrino with $p_{\mathrm{T}}>25 \mathrm{GeV}$ originating from a $W$-boson decay (with $m_{\mathrm{T}}^{W}>60 \mathrm{GeV}$ ) and one or two hadron level jets with $p_{\mathrm{T}}>25 \mathrm{GeV}$ and $|\eta|<2$. 1 . At least one of the jets is required to be a $b$-jet, defined by the presence of a weakly decaying $b$-hadron with $p_{\mathrm{T}}>5 \mathrm{GeV}$ and within $\Delta R \leq 0.3$ of the jet axis.

At reconstruction level, events are required to contain a $W$-boson candidate and either one or two reconstructed jets of which exactly one is tagged as a $b$-jet. The $b$ tagging algorithm used in this analysis exploits the $b$-quark lifetime and decay topologies to discriminate $b$-jets from $c$-jets and light jets. The measurement of secondary vertices and impact parameters of tracks inside jets are combined using an artificial neural network to determine a single discriminant output [18]. The working point used for the selection corresponds to a $b$-tagging efficiency of about $40 \%$ at low $p_{\mathrm{T}}$, increasing to a plateau of $57 \%$ for $b$-jets with $p_{\mathrm{T}}$ above $60 \mathrm{GeV}$, with rejection rates of about 10 for $c$-jets and 1000 for light-jets.

The measurement of $W+b$ production is very challenging due to the large background which amounts to $85 \%$ of the events passing the signal selection. The singletop, $t \bar{t}$ and multi-jet contributions are estimated either in background-enriched control regions or directly in the signal regions using kinematic distributions. The $W+b$-jets, $W+c$-jets and $W+$ light-jets contributions are then statistically separated, and the number of $W+b$-jets events is extracted, by fitting the $b$-tagging discriminant distribution of $b$-tagged jets in data. For more information on the analysis the reader is referred to Ref. [5].

An unfolding procedure is applied to correct the measured $W+b$ yields to the fiducial region of the measurement to calculate the fiducial cross sections. The cross sections measured in the 1-jet and 2-jet fiducial regions and in the combined 1+2-jet fiducial region are:

$$
\begin{aligned}
\sigma(W b, 1 \text { jet }) & =5.0 \pm 0.5(\text { stat }) \pm 1.2(\text { syst }) \mathrm{pb} \\
\sigma(W b, 2 \text { jets }) & =2.2 \pm 0.2(\text { stat }) \pm 0.5(\text { syst }) \mathrm{pb} \\
\sigma(W b, 1+2 \text { jets }) & =7.1 \pm 0.5(\text { stat }) \pm 1.4(\text { syst }) \mathrm{pb} .
\end{aligned}
$$

The systematic uncertainty of the measurement in the $1+2$-jet bin amounts to $20 \%$ and is dominated by the uncertainty on the jet energy scale and resolution. The measured cross sections are compared to theoretical predictions in Figure 3. The results are compared to the NLO predictions of MCFM [19] and PowHEg [20], and to the predictions of ALPGEN interfaced to HERWIG and JIMMY scaled by the NNLO normalization factor for the 


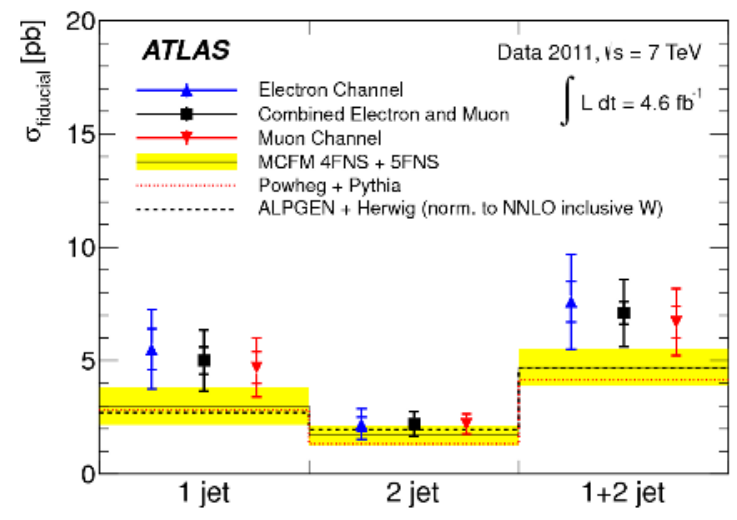

Figure 3: Measured fiducial $W+b$-jets cross sections with the statistical (inner error bar) and statistical plus systematic (outer error bar) uncertainties [5]. The measurements are compared with NLO predictions calculated with MCFM and corrected for hadronization and DPI effects. The yellow bands represent the total uncertainty on the prediction (quadratic sum of uncertainties due to scale, PDF, DPI model and non-perturbative corrections). The NLO prediction from PowHEg interfaced to PYTHIA, corrected for DPI effects, and the prediction from ALPGEN interfaced to HERWIG and scaled by the NNLO inclusive $W$ normalization factor are also shown.

inclusive $W$ cross section [17]. To compare the NLO calculations with data, corrections for non-perturbative effects are applied and the effect of DPI, which represents a $25 \%$ effect on the total cross section, is considered.

In the 1-jet fiducial region, the measured fiducial cross section is larger than the NLO predictions, but compatible within the theoretical and experimental uncertainties. The measurement is in good agreement with the prediction in the 2-jets bin. The cross section as a function of the $b$-jet $p_{\mathrm{T}}$ is measured in the range between $25 \mathrm{GeV}$ and $140 \mathrm{GeV}$ [5]. The theoretical predictions provide a good description of the data at low $b$-jet $p_{\mathrm{T}}(<40 \mathrm{GeV})$, but deviates increasingly from the measured $W+b$ production rate at higher $p_{\mathrm{T}}$. The measured spectrum is harder than the prediction. A second set of measurements, including the single-top contribution, is also performed. The corresponding $b$-jet $p_{\mathrm{T}}$ differential cross sections have significantly reduced uncertainties with respect to the single-topsubtracted measurements and can be compared to combined single top-quark and $W+b$-jets calculations in the future.

\section{$5 \mathrm{~W}+\mathrm{c}$}

The $p p \rightarrow W c+X$ production process is sensitive to the $s$-quark distribution function in the proton, at values of the momentum transfer of the order of the mass of the $W$ boson. The single-charm production process can be isolated from $p p \rightarrow W c \bar{c}+X$ production by exploiting the charge

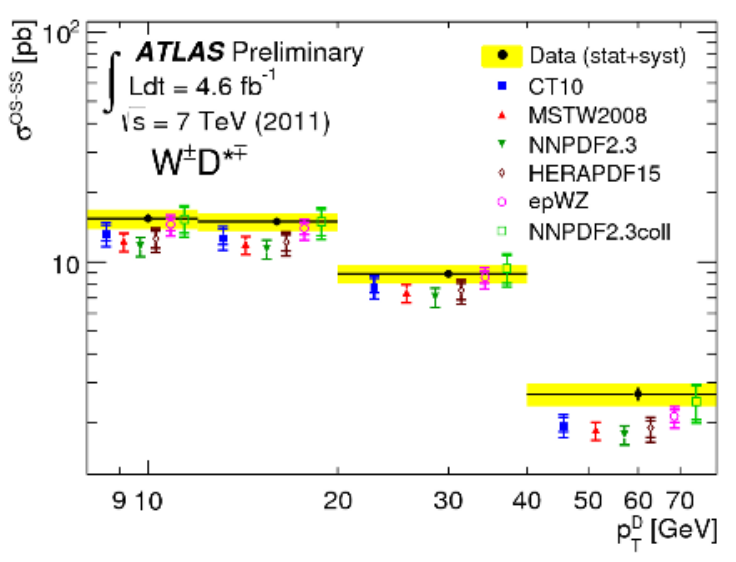

(a)

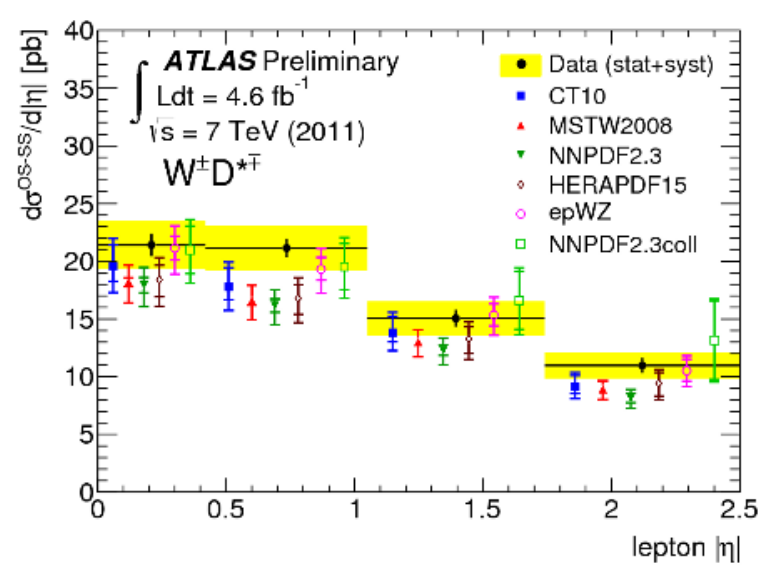

(b)

Figure 4: Measured differential cross sections $\sigma\left(W^{ \pm} D^{* \mp}\right)$ as a function of (a) $p_{\mathrm{T}}^{D}$ and (b) $\left|\eta^{\ell}\right|$ compared to predictions obtained using different PDF sets [6]. The measurement is shown together with the statistical and systematical uncertainties. The inner error bars on the theoretical predictions show the $68 \%$ C.L. uncertainties obtained from the PDF error sets, while the outer error bars represent the total theoretical uncertainty (quadratic sum of PDF uncertainty, scale uncertainty and fragmentation uncertainty).

correlation between the $W$ and the charm quark. This approach is pursued in the ATLAS measurement of $W+c$ production presented here [6]. The measurement is based on a data set corresponding to an integrated luminosity of $4.6 \mathrm{fb}^{-1}$.

In events where a $W$ boson decays to an electron or muon, charmed mesons are reconstructed using the decay modes $D^{+} \rightarrow K^{-} \pi^{+} \pi^{+}$and $D^{*+} \rightarrow D^{0} \pi^{+}$with $D^{0} \rightarrow K^{-} \pi^{+}, D^{0} \rightarrow K^{-} \pi^{+} \pi^{0}$ or $D^{0} \rightarrow K^{-} \pi^{+} \pi^{-} \pi^{+}$, and their charge conjugates. Distributions in which the charm hadron and the $W$ boson have opposite charge (OS) and where they have the same charge (SS) are formed. The single-charm yield is then extracted by fitting the mass (for the $D^{+}$) or mass difference $\Delta m=m\left(D^{*}\right)-m\left(D^{0}\right)$ (for the $D^{*+}$ ) distribution in OS minus SS events. The resulting 
$W^{ \pm} D^{(*) \mp}$ yields are corrected for detector effects and for their relative branching ratios [21], resulting in one measurement of the $D^{+}$spectrum and three measurements of the $D^{(*)+}$ spectra, where the latter are combined in the final result.

The fiducial cross sections $\sigma\left(W^{ \pm} D^{(*) \mp}\right)$ are measured inclusively and differentially as a function of the $D$ hadron transverse momentum and as a function of the pseudorapidity of the lepton from the $W$ decay. The fiducial region of the measurement is defined by the lepton transverse momentum $p_{\mathrm{T}}^{\ell}>20 \mathrm{GeV}$ and pseudorapidity $\left|\eta^{\ell}\right|<2.5$, neutrino transverse momentum $p_{\mathrm{T}}^{v}>25 \mathrm{GeV}, W$ transverse mass $m_{\mathrm{T}}^{W}>40 \mathrm{GeV}$ and charm hadron $\left(D^{*+}\right.$ or $\left.D^{+}\right)$ transverse momentum $p_{T}^{D}>8 \mathrm{GeV}$ and pseudorapidity $\left|\eta^{D}\right|<2.2$.

The measured differential cross sections in the $D^{*+}$ channel are shown in Figure 4. The predictions are obtained using the aMC@NLO simulation [22], which is based on the MC@NLO formalism and the MADGRAPH5 framework [23], with Herwig++ [24] used to model the parton-shower, hadronization and underlying-event. The events are generated with different NLO and NNLO PDF sets that differ in their strange quark parameterizations: CT10 [25], MSTW2008 [26], NNPDF2.3 and NNPDF2.3coll [27], HERAPDF15 [28] and epWZ [29]. For MSTW2008, HERAPDF15 and NNPDF2.3, the $s$ quark sea is suppressed relative to the $d$-quark for all values of $x$. The strange sea in CT10 is less suppressed than in MSTW2008 or NNPDF2.3. NNPDF2.3coll and epWZ PDF sets are determined based on collider data only and have a comparable $s$-quark sea and $d$-quark sea.

The shapes of the $p_{\mathrm{T}}^{D}$ and $\left|\eta^{\ell}\right|$ distributions for the different PDF sets are similar, but the predicted cross sections differ by as much as $25 \%$. The measured integrated cross sections in OS-SS events are

$$
\begin{aligned}
\sigma\left(W^{ \pm} D^{* \mp}\right) & =42.4 \pm 1.0(\text { stat }) \pm 3.6(\text { syst }) \mathrm{pb} \\
\sigma\left(W^{ \pm} D^{\mp}\right) & =41.9 \pm 2.2(\text { stat }) \pm 3.2(\text { syst }) \mathrm{pb} .
\end{aligned}
$$

The uncertainty of the measurement is dominated by the systematic uncertainty which arises mainly from the uncertainty on the tracking efficiency. The measurements agree well with the predictions obtained with the epWZ and NNPDF2.3coll PDF sets and lie about 1.1(1.7) $\sigma$ for $D^{*+}\left(D^{+}\right)$above the central value of the CT10 PDF. Somewhat larger discrepancies are seen with MSTW2008, HERAPDF15 and NNPDF2.3. These results favor PDFs where the $s$-quark and $d$-quark sea contributions are comparable at $x \sim 0.01$.

\section{Conclusion}

The high-statistics data samples at a center-of-mass energy of $7 \mathrm{TeV}$ collected with the ATLAS experiment allow for high precision measurements of vector boson plus jets production up to high jet multiplicities in a large kinematic phase space. In general, the NLO pQCD predictions provide a good description of the measured data. However, some tensions are observed in the comparison of kinematic distributions at large transverse momenta. These are attributed to missing higher order corrections in the calculation or to the matching scheme and are expected to be improved in the future.

The production of $W+2$-jet events has been analyzed to derive information about the amount of DPI in protonproton collisions at $\sqrt{s}=7 \mathrm{TeV}$. The measurement of $\sigma_{\text {eff }}$ is consistent with previous measurements at lower energies.

The cross sections of vector boson plus heavy flavor production are found to be in good agreement with theoretical prediction. The measurement of $W+c$ production shows sensitivity to the strange content of the proton and favors PDF sets which have a comparable $d$-quark and $s$ quark sea.

\section{References}

[1] ATLAS Collaboration, JINST 3 S08003 (2008)

[2] ATLAS Collaboration, Phys. Rev. D85 092002 (2012)

[3] ATLAS Collaboration, arXiv:1304.7098 (2013), accepted by JHEP

[4] ATLAS Collaboration, New J. Phys. 15033038 (2013)

[5] ATLAS Collaboration, arXiv:1302.2929 (2013), accepted by JHEP

[6] ATLAS Collaboration, ATLAS-CONF-2013-045, http://cds.cern.ch/record/1546800

[7] ATLAS Collaboration, Phys. Lett. B706 295-313 (2012)

[8] C. F. Berger et al., Phys. Rev. D78 036003 (2008)

[9] C. F. Berger et al., Phys. Rev. D82 074002 (2010)

[10] H. Ita et al., Phys. Rev. D85 031501 (2012)

[11] T. Gleisberg et al., JHEP 0902007 (2009)

[12] M. L. Mangano et al., JHEP 07001 (2003)

[13] G. Corcella et al., JHEP 01010 (2001)

[14] J. M. Butterworth et al., Z. Phys. C72 637 (1996)

[15] S. Hoeche et al., JHEP 04027 (2013)

[16] S. Frixione and B. R. Webber, JHEP 06029 (2002)

[17] C. Anastasiou et al., Phys. Rev. D69 094008 (2004)

[18] ATLAS Collaboration, ATLAS-CONF-2011-102, http://cds.cern.ch/record/1369219

[19] J. M. Campbell et al., Phys. Rev. D86 034021 (2012)

[20] S. Alioli et al., JHEP 06043 (2010)

[21] J. Beringer et al. (Particle Data Group), Phys. Rev. D86, 010001 (2012)

[22] R. Frederix et al., Phys. Lett. B701 427-433 (2011)

[23] J. Alwall et al., JHEP 06128 (2011)

[24] M. Bahr et al., Eur. Phys. J. C58 639-707 (2008)

[25] H.-L. Lai et al., Phys. Rev. D82 074024 (2010)

[26] A. D. Martin et al., Eur. Phys. J. C63 189-285 (2009)

[27] R. D. Ball et al., Nucl. Phys. B809 1-63 (2009)

[28] H1 and ZEUS Collaborations, JHEP 01109 (2010)

[29] ATLAS Collaboration, Phys. Rev. Lett. 109012001 (2012) 